PROCEEDINGS OF THE

AMERICAN MATHEMATICAL SOCIETY

Volume 125, Number 1, January 1997, Pages 51-60

S 0002-9939(97)03880-X

\title{
TRANSITIVE FACTORISATIONS INTO TRANSPOSITIONS AND HOLOMORPHIC MAPPINGS ON THE SPHERE
}

\author{
I. P. GOULDEN AND D. M. JACKSON
}

(Communicated by Jeffry N. Kahn)

\begin{abstract}
We determine the number of ordered factorisations of an arbitrary permutation on $n$ symbols into transpositions such that the factorisations have minimal length and such that the factors generate the full symmetric group on $n$ symbols. Such factorisations of the identity permutation have been considered by Crescimanno and Taylor in connection with a class of topologically distinct holomorphic maps on the sphere. As with Macdonald's construction for symmetric functions that multiply as the classes of the class algebra, essential use is made of Lagrange inversion.
\end{abstract}

\section{INTRODUCTION}

Let $\mathcal{C}_{\alpha}$ be the conjugacy class of the symmetric group $\mathfrak{S}_{n}$ on $n$ symbols, indexed by the partition $\alpha$ of $n$, for $n \geq 1$, denoted by $\alpha \vdash n$, and let $h^{\alpha}$ be the size of this conjugacy class. Let $\mathrm{K}_{\alpha}$ be the sum in $\mathbb{C S}_{n}$ of all elements of $\mathcal{C}_{\alpha}$, for $\alpha \vdash n$. The number of parts of $\alpha$ is denoted by $l(\alpha)$.

The coefficient of $\mathrm{K}_{\alpha}$ in the product $\mathrm{K}_{\beta} \mathrm{K}_{\gamma}$, for $\alpha, \beta, \gamma \vdash n$, is called the connection coefficient in the class algebra of $\mathfrak{S}_{n}$. Up to a constant depending on $h^{\alpha}, h^{\beta}$ and $h^{\gamma}$, it is the number of ordered factorisations $\left(\sigma_{1}, \sigma_{2}\right)$ of $\pi \in \mathcal{C}_{\alpha}$ with factors $\sigma_{1}, \sigma_{2}$ in $\mathcal{C}_{\beta}$ and $\mathcal{C}_{\gamma}$, respectively. If the condition

$$
(n-l(\alpha)) \leq(n-l(\beta))+(n-l(\gamma))
$$

fails, then the connection coefficient is zero. Such factorisations arise in a number of topological contexts, such as the determination of the Euler characteristic for the moduli space of curves $[5,8]$ and, more generally, the enumeration of two-cell embeddings [9].

In general, the connection coefficient can be expressed as a character sum, and this has been exploited (see, e.g. $[9,10]$ ) to address combinatorial questions that can be expressed in terms of certain connection coefficients. However, the determination of this character sum is difficult in most instances, so other approaches to connection coefficients are desirable. One such approach is given in [6], to evaluate the connection coefficient in the case that

$$
(n-l(\alpha))=(n-l(\beta))+(n-l(\gamma)) .
$$

These are called top coefficients, since partitions related by this equality are on the boundary of the region where the coefficient is zero. A construction in [6]

Received by the editors July 20, 1995.

1991 Mathematics Subject Classification. Primary 05A15; Secondary 05E99, 58C10, 70H20.

(C)1997 American Mathematical Society 
uses the compositional inverse of the generating series for the complete symmetric functions to produce a basis for symmetric functions whose connection coefficients in the ring of symmetric functions are precisely the top connection coefficients, after reindexing. The results obtained thus require the use of Lagrange inversion in a fundamental way, to carry out this compositional inversion. The combinatorial setting for this is embeddings of vertex 2-coloured trees in the sphere, a setting that is suggestive of constructions for other connection coefficients that are close to top.

Recently, an interesting new group theoretic restriction on factorisations, arising from another topological context, has been encountered by Crescimanno and Taylor [1]. Their work involves ordered factorisations of the identitity permutation into $2 n-2$ transpositions, with the additional condition that the factors generate $\mathfrak{S}_{n}$. They obtain the surprising result that the number of such factorisations is

$$
(2 n-2) ! n^{n-3} \text {. }
$$

Their interest in this problem arose from the fact that it equals $n$ ! times the number of topologically distinct holomorphic maps from $S^{2}$ to $S^{2}$ with $2 n-2$ elementary branch point singularities whose images are fixed.

It is readily seen that $2 n-2$ is the minimal number of transpositions whose product can equal the identity under the restriction that the transpositions generate $\mathfrak{S}_{n}$. We can state the analogous question for an arbitrary permutation in the following way: Let $c_{\alpha}$ be the number of $j$-tuples of permutations $\left(\sigma_{1}, \ldots, \sigma_{j}\right)$ such that, for an arbitrary but fixed $\pi \in \mathcal{C}_{\alpha}$,

(a) $\pi=\sigma_{1} \cdots \sigma_{j}$

(b) $\sigma_{1}, \ldots, \sigma_{j} \in \mathcal{C}_{\left[21^{n-2}\right]}$,

(c) $\sigma_{1}, \ldots, \sigma_{j}$ generate $\mathfrak{S}_{n}$,

(d) $j$ is minimal, subject to (a), (b), (c).

We call $\left(\sigma_{1}, \ldots, \sigma_{j}\right)$ a minimal, transitive, ordered factorisation of $\pi$ into transpositions. (In this notation, Crescimanno and Taylor showed that $c_{\left[1^{n}\right]}=(2 n-2) ! n^{n-3}$.) It is shown in Section 2 that the minimal choice of $j$ in condition (d) is $j=\mu(\alpha)$, where

$$
\mu(\alpha)=n+l(\alpha)-2 .
$$

Note that, in the case that $\pi \in \mathcal{C}_{[n]}$, condition (c) is forced by condition (a), so this problem is simply the "top" problem solved in [6]; in particular Dénes [2] first showed that $c_{[n]}=n^{n-2}$.

The main result of this paper is the following generalisation of Crescimanno and Taylor's and Dénes' results, giving an explicit expression for $c_{\alpha}$ when $\alpha$ is arbitrary.

Theorem 1.1. Let $\alpha=\left(\alpha_{1}, \alpha_{2}, \ldots, \alpha_{k}\right) \vdash n$, for $n, k \geq 1$. Then

$$
c_{\alpha}=n^{k-3}(n+k-2) ! \prod_{j=1}^{k} \frac{\alpha_{j}^{\alpha_{j}}}{\left(\alpha_{j}-1\right) !} .
$$

Our method is to determine a differential equation that a generating series for $c_{\alpha}$ satisfies. This is given in Section 2. In Section 3 we prove that the corresponding series for the numbers given in Theorem 1.1 also satisfies the equation, and check initial conditions so this is unique. This latter series is manipulated in terms of two functional equations by Lagrange inversion, which seems essential to provide tractable expressions for the quantities required. However, we have not found the analogous construction to that for top coefficients, in which a compositional inverse 
was an essential part of the construction from the outset. A character theoretic expression for $c_{\alpha}$ is given in Section 4, although we have not been able to deduce Theorem 1.1 from this expression.

\section{A COMbinatorial CONSTRUCtion}

We begin with an analysis of multiplication by a transposition. For $\pi \in \mathfrak{S}_{n}$ let $\kappa(\pi)$ denote the number of cycles in (the disjoint cycle representation of) $\pi$, and let $\sigma$ be the transposition $(a, b)$. We now consider separately two cases for the product $\pi \sigma$. If $a$ and $b$ are on different cycles of $\pi$, then the cycles of $\pi$ containing elements $a$ and $b$ are joined together in $\pi \sigma$, so $\kappa(\pi \sigma)=\kappa(\pi)-1$, and in this case we call $\sigma$ a join for $\pi$. If $a$ and $b$ are on the same cycle of $\pi$, then the cycle of $\pi$ containing $a$ and $b$ is cut into two cycles in $\pi \sigma$, one containing $a$ and the other containing $b$, so $\kappa(\pi \sigma)=\kappa(\pi)+1$, and in this second case we call $\sigma$ a cut for $\pi$. Now suppose that $\left(\sigma_{1}, \ldots, \sigma_{j}\right)$ is an ordered factorisation of $\pi$ into transpositions (i.e., it satisfies conditions (a) and (b) above, but not necessarily (c) or (d)), and that $r$ of the transpositions are joins and $s$ are cuts (as they are multiplied together from left to right). Then by induction, starting with the identity permutation having $n$ cycles, and using the relationships for $\kappa$ in the two cases above, we have

$$
\kappa(\pi)=n-r+s .
$$

Proposition 2.1. If $\alpha=\left(\alpha_{1}, \alpha_{2}, \ldots\right) \vdash n$, for $n \geq 1$, then $\mu(\alpha)=n+l(\alpha)-2$.

Proof. Suppose $\left(\sigma_{1}, \ldots, \sigma_{j}\right)$ is a transitive, ordered factorisation of $\pi \in \mathcal{C}_{\alpha}$ into transpositions (i.e., it satisfies conditions (a), (b), (c) above, but not necessarily (d)). Let $G\left(\sigma_{1}, \ldots, \sigma_{j}\right)$ be the graph on vertices labelled $\{1, \ldots, n\}$, and edges labelled $\{1, \ldots, j\}$ in which the edge labelled $i$ joins the vertices interchanged by the transposition $\sigma_{i}$, for $i=1, \ldots, j$.

Condition (c) means that $G=G\left(\sigma_{1}, \ldots, \sigma_{j}\right)$ is connected. Let $T$ be the spanning tree of $G$ formed by, for $i=1, \ldots, j$, selecting the edge labelled $i$ for $T$ if and only if it joins vertices in two different components of $G\left(\sigma_{1}, \ldots, \sigma_{i-1}\right)$ (this is Kruskal's algorithm with the edges ordered by their labels). Now $T$ contains $n-1$ edges, all of whose corresponding transpositions must be joins, by construction. Of the edges of $G$ that are not in $T$, suppose that $g$ are joins and $h$ are cuts. Then from (2) we obtain $\kappa(\pi)=n-(n-1+g)+h$. But the number of edges of $G$ is $n-1+g+h$, and $\kappa(\pi)=l(\alpha)$, so

$$
j=n-1+g+h=n+l(\alpha)-2+2 g \geq n+l(\alpha)-2,
$$

with equality when $g=0$ (and thus $h=l(\alpha)-1$ ). This can be achieved for any $\alpha$ by, for instance, choosing the first $n-1$ edges of $G$ as the edges of any tree on vertices $1, \ldots, n$, in any order (so these are all joins); thus we will have $\sigma_{1} \cdots \sigma_{n-1} \in \mathcal{C}_{[n]}$. Then choose $h=l(\alpha)-1$ edges to cut this cycle successively into cycles of lengths $\alpha_{1}, \alpha_{2}, \ldots$, so we have $\sigma_{1} \cdots \sigma_{n+l(\alpha)-2} \in \mathcal{C}_{\alpha}$. Any required $\pi \in \mathcal{C}_{\alpha}$ can be obtained in this way by suitably relabelling the vertices of $G$.

The analysis above can be extended to determine the cycle distribution of permutations in the product $\pi \mathrm{K}_{\left[21^{n-2}\right]}$ by means of a differential operator as follows. Let $\mathbf{p}=\left(p_{1}, p_{2}, \ldots\right)$ be indeterminates, and $p_{\lambda}=p_{\lambda_{1}} p_{\lambda_{2}} \ldots$, for $\lambda=\left(\lambda_{1}, \lambda_{2}, \ldots\right)$, a partition. If $\tau(\pi) \vdash n$ is the partition listing the lengths of the cycles of $\pi$, and we 
define $\Phi(\pi)=p_{\tau(\pi)}$, extended linearly to the whole of $\mathbb{C S}_{n}$, then, as is considered in $[4]$,

where

$$
\Phi\left(\pi \mathrm{K}_{\left[21^{n-2}\right]}\right)=\frac{1}{2}(J+C) \Phi(\pi)
$$

$$
J=\sum_{i, j \geq 1} p_{i+j} i j \frac{\partial^{2}}{\partial p_{i} \partial p_{j}}, \quad C=\sum_{i, j \geq 1} p_{i} p_{j}(i+j) \frac{\partial}{\partial p_{i+j}} .
$$

The first operator $J$ gives the contribution from the transpositions that are joins for $\pi$; the summand corresponding to $i$ and $j$ joins together an $i$-cycle and a $j$-cycle. The second operator $C$ gives the contribution from the transpositions that are cuts for $\pi$; the summand corresponding to $i$ and $j$ cuts an $i+j$-cycle into an $i$-cycle and a $j$-cycle. (In the case that $p_{i}$ is regarded as the $i$-th power sum symmetric function, then $J$ and $C$ are mutually adjoint operators on the ring of symmetric functions, equipped with the usual inner product.)

We now use this combinatorial apparatus to determine an equation for the required numbers $c_{\alpha}$.

Lemma 2.2. The generating series

$$
F=\sum_{n \geq 1} \sum_{\alpha \vdash n} c_{\alpha} \frac{z^{n}}{n !} \frac{h^{\alpha}}{\mu(\alpha) !} p_{\alpha}
$$

satisfies the differential equation

$$
L(F)=0,
$$

where

$$
L(F)=\frac{1}{2} \sum_{i, j \geq 1}\left(p_{i+j} i \frac{\partial F}{\partial p_{i}} j \frac{\partial F}{\partial p_{j}}+(i+j) p_{i} p_{j} \frac{\partial F}{\partial p_{i+j}}\right)-z \frac{\partial F}{\partial z}-\sum_{i \geq 1} p_{i} \frac{\partial F}{\partial p_{i}}+2 F .
$$

Proof. Let $\widetilde{F}$ be the generating series for minimal, transitive, ordered factorisations $\left(\sigma_{1}, \ldots, \sigma_{j}\right)$ of permutations $\pi$ in $\bigcup_{n>1} \mathfrak{S}_{n}$, in which $p_{i}$ is an ordinary marker for cycles of length $i$ in $\pi$, for $i \geq 1$, and, in the terminology of the proof of Proposition 2.1, $z$ is an exponential marker for the vertices in $G$, and $u$ is an exponential marker for the edges in $G$. Thus

$$
\widetilde{F}=\sum_{n \geq 1} \sum_{\alpha \vdash n} c_{\alpha} \frac{z^{n}}{n !} \frac{u^{\mu(\alpha)}}{\mu(\alpha) !} h^{\alpha} p_{\alpha}=\sum_{n \geq 1} \sum_{\alpha \vdash n} c_{\alpha} \frac{z^{n}}{n !} \frac{u^{\mu(\alpha)}}{\mu(\alpha) !} \Phi\left(\mathrm{K}_{\alpha}\right) .
$$

Consider a minimal, transitive, ordered factorisation $\left(\sigma_{1}, \ldots, \sigma_{j}\right)$ of $\pi \in \mathfrak{S}_{n}$ into transpositions. Suppose we remove the edge of largest label (corresponding to transposition $\sigma_{j}$ ) from $G$, without changing $\pi$. This modifies the generating series to become

$$
\frac{\partial \widetilde{F}}{\partial u}
$$

This may be determined in another way, by considering two cases for $\sigma_{j}$. If $\sigma_{j}$ corresponds to a non-tree edge, then it is a cut, and thus $\left(\sigma_{1}, \ldots, \sigma_{j-1}\right)$ is a minimal, transitive, ordered factorisation in $\mathfrak{S}_{n}$, so the contribution to the modified generating series from this case is

$$
C \widetilde{F} \text {. }
$$

Otherwise, if $\sigma_{j}$ corresponds to a tree edge, then it is a join consisting of vertices from each of the exactly two components in $G\left(\sigma_{1}, \ldots, \sigma_{j-1}\right)$, and each of these 
components corresponds to a minimal, transitive, ordered factorisation in the symmetric group on its vertex set. Thus the contribution to the modified generating series from this case is

$$
\frac{1}{2} \sum_{i, j \geq 1} p_{i+j}\left(i \frac{\partial \widetilde{F}}{\partial p_{i}}\right)\left(j \frac{\partial \widetilde{F}}{\partial p_{j}}\right),
$$

using the multiplication rule for exponential generating series, with both vertices and edges labelled, since we are joining cycles in the two components.

Thus $\widetilde{F}$ satisfies the differential equation

$$
\frac{1}{2} \sum_{i, j \geq 1}\left(p_{i+j} i \frac{\partial \widetilde{F}}{\partial p_{i}} j \frac{\partial \widetilde{F}}{\partial p_{j}}+(i+j) p_{i} p_{j} \frac{\partial \widetilde{F}}{\partial p_{i+j}}\right)=\frac{\partial \widetilde{F}}{\partial u} .
$$

But $\widetilde{F}$ evaluated at $u=1$ is $F$, and

$$
\left.\frac{\partial \widetilde{F}}{\partial u}\right|_{u=1}=z \frac{\partial F}{\partial z}+\sum_{i \geq 1} p_{i} \frac{\partial F}{\partial p_{i}}-2 F,
$$

so this differential equation can be rewritten in terms of $F$, giving the result.

\section{Proof of the Main Result}

In this section we establish Theorem 1.1 by proving that

$$
G=\sum_{n \geq 1} \sum_{\alpha \vdash n} n^{l(\alpha)-3}\left(\prod_{j \geq 1} \frac{\alpha_{j}^{\alpha_{j}}}{\left(\alpha_{j}-1\right) !}\right) h^{\alpha} p_{\alpha} \frac{z^{n}}{n !}
$$

is the unique choice for the series $F$ considered in the previous section. Our proof consists of a verification that $G$ satisfies the differential equation $z \frac{\partial}{\partial z} L(G)=0$, which $F$ also satisfies, by Lemma 2.2. Uniqueness is then established via initial conditions. Two technical results are needed for this verification. Both use Lagrange's Implicit Function Theorem ([7], Section 1.2) in an essential way.

The first of these technical results concerns the functional equation

$$
s=z \exp \left(\sum_{i \geq 1} \frac{i^{i}}{i !} p_{i} s^{i}\right)
$$

and expresses each term that arises in $z \frac{\partial}{\partial z} L(G)$ in terms of the unique solution $s \equiv s(z, \mathbf{p})$ to this equation.

\section{Proposition 3.1.}

1.

$$
\left(z \frac{\partial}{\partial z}\right)^{2} G=\sum_{i \geq 1} \frac{i^{i}}{i !} p_{i} s^{i}
$$

2.

$$
z \frac{\partial G}{\partial z}=\sum_{i \geq 1} \frac{i^{i-1}}{i !} p_{i} s^{i}-\frac{1}{2}\left(\sum_{i \geq 1} \frac{i^{i}}{i !} p_{i} s^{i}\right)^{2}
$$


3.

$$
z \frac{\partial^{2} G}{\partial z \partial p_{k}}=\frac{k^{k-1}}{k !} s^{k}, \quad \text { for } k \geq 1,
$$

4.

$$
\frac{\partial G}{\partial p_{k}}=\frac{k^{k-2}}{k !} s^{k}-\frac{k^{k-1}}{k !} \sum_{i \geq 1} \frac{i^{i+1}}{i !} p_{i} \frac{s^{k+i}}{k+i}, \quad \text { for } k \geq 1 .
$$

Proof. 1. From (5), with $[A] B$ denoting "the coefficient of $A$ in $B$ ", we have

$$
G=\sum_{n \geq 1} \frac{1}{n^{3}} z^{n}\left[\lambda^{n}\right] \exp \left(n \sum_{i \geq 1} \frac{i^{i}}{i !} p_{i} \lambda^{i}\right) .
$$

Then Lagrange's Theorem applied to (6) gives

$$
G=\sum_{n \geq 1} \frac{1}{n^{2}} z^{n}\left\{\frac{1}{n}\left[\lambda^{n-1}\right] \frac{1}{\lambda}\left(\exp \sum_{i \geq 1} \frac{i^{i}}{i !} p_{i} \lambda^{i}\right)^{n}\right\}=\sum_{n \geq 1} \frac{1}{n^{2}} z^{n}\left[z^{n}\right] \log \left(\frac{s}{z}\right),
$$

and the result follows immediately.

2. Differentiating (6) partially with respect to $z$, and solving for $\frac{\partial s}{\partial z}$, we obtain

$$
z \frac{\partial s}{\partial z}=s\left(1-\sum_{i \geq 1} \frac{i^{i+1}}{i !} p_{i} s^{i}\right)^{-1} .
$$

But part 1 of this proposition gives

$$
z \frac{\partial G}{\partial z}=\int_{0}^{z} \frac{1}{z}\left(\sum_{i \geq 1} \frac{i^{i}}{i !} p_{i} s^{i}\right) d z=\int_{0}^{z}\left(\sum_{i \geq 1} \frac{i^{i}}{i !} p_{i} s^{i-1}\right)\left(1-\sum_{i \geq 1} \frac{i^{i+1}}{i !} p_{i} s^{i}\right) \frac{\partial s}{\partial z} d z
$$

from (8). Now change the variable of integration to $s$, giving

$$
z \frac{\partial G}{\partial z}=\int_{0}^{s}\left(\sum_{i \geq 1} \frac{i^{i}}{i !} p_{i} s^{i-1}\right)\left(1-\sum_{i \geq 1} \frac{i^{i+1}}{i !} p_{i} s^{i}\right) d s,
$$

and the result follows.

3. Differentiating (6) partially with respect to $p_{k}$ and solving for $\frac{\partial s}{\partial p_{k}}$, we have, for $k \geq 1$,

$$
\frac{\partial s}{\partial p_{k}}=\frac{k^{k}}{k !} s^{k+1}\left(1-\sum_{i \geq 1} \frac{i^{i+1}}{i !} p_{i} s^{i}\right)^{-1}
$$

Comparing this expression with (8), we obtain

$$
\frac{\partial s}{\partial p_{k}}=\frac{k^{k}}{k !} s^{k} z \frac{\partial s}{\partial z} .
$$


Thus, from (7),

$$
\begin{aligned}
z \frac{\partial^{2} G}{\partial z \partial p_{k}} & =\sum_{n \geq 1} \frac{1}{n} z^{n}\left[z^{n}\right] \frac{1}{s} \frac{\partial s}{\partial p_{k}}=\sum_{n \geq 1} \frac{1}{n} z^{n}\left[z^{n-1}\right] \frac{k^{k}}{k !} s^{k-1} \frac{\partial s}{\partial z} \\
& =\sum_{n \geq 1} \frac{1}{n} z^{n}\left[z^{n-1}\right] \frac{\partial}{\partial z}\left(\frac{k^{k-1}}{k !} s^{k}\right),
\end{aligned}
$$

and the result follows.

4. Part 3 of this proposition gives

$$
\frac{\partial G}{\partial p_{k}}=\frac{k^{k-1}}{k !} \int_{0}^{z} \frac{s^{k}}{z} d z=\frac{k^{k-1}}{k !} \int_{0}^{s} s^{k-1}\left(1-\sum_{i \geq 1} \frac{i^{i+1}}{i !} p_{i} s^{i}\right) d s,
$$

from (8), changing the variable of integration to $s$. The result follows.

The second of these technical results concerns the functional equation $w=z e^{w}$, and the evaluation of two Abel-type summations that arise in our verification. The first of these results is straightforward, but the second seems more difficult and, as far as we can tell, is new.

Proposition 3.2. 1. For $m \geq 1$, let $S_{m}=\sum_{\substack{i, j \geq 1 \\ i+j=m}} \frac{i^{i}}{i !} \frac{j^{j-1}}{j !}$. Then

$$
S_{m}=\frac{m^{m}}{m !}-\frac{m^{m-1}}{m !} .
$$

2. For $k, m \geq 1$, let $T_{k, m}=\frac{k^{k+1}}{k !} \sum_{\substack{i \geq 1, j \geq 0 \\ i+j=m}} \frac{i^{i}}{i !} \frac{j^{j}}{j !} \frac{1}{k+j}$. Then

$$
T_{k, m}+T_{m, k}=\frac{(k+m)^{k+m}}{(k+m) !} .
$$

Proof. 1. By Lagrange's Theorem,

$$
w=\sum_{i \geq 1} \frac{i^{i-1}}{i !} z^{i} \quad \text { and } \quad \frac{w}{1-w}=z \frac{d w}{d z}=\sum_{i \geq 1} \frac{i^{i}}{i !} z^{i} .
$$

Thus, for $m \geq 1$,

$$
S_{m}=\left[z^{m}\right] \frac{w}{1-w} w=\left[z^{m}\right]\left(\frac{w}{1-w}-w\right)
$$

and the result follows from (9).

2. Let $u=w(x)$ and $v=w(y)$ for $w$ given above, so $u=x e^{u}$ and $v=y e^{v}$. Then

$$
\begin{aligned}
T(x, y) & =\sum_{k, m \geq 1} T_{k, m} x^{k} y^{m}=\sum_{k \geq 1} \frac{k^{k+1}}{k !} x^{k}\left(\sum_{i \geq 1} \frac{i^{i}}{i !} y^{i}\right)\left(\sum_{j \geq 0} \frac{j^{j}}{j !} \frac{y^{j}}{j+k}\right) \\
& =\sum_{k \geq 1} \frac{k^{k+1}}{k !} x^{k} \frac{v}{1-v} \frac{1}{y^{k}} \int_{0}^{y} \frac{y^{k-1}}{1-v} d y,
\end{aligned}
$$


from (9). But, from (9) and using the functional equation for $v$ to change the variable of integration, we obtain

$$
\begin{aligned}
\int_{0}^{y} & \frac{y^{k-1}}{1-v} d y=\int_{0}^{v} v^{k-1} e^{-k v} d v=k^{-k} \int_{0}^{k v} t^{k-1} e^{-t} d t \\
& =\frac{k !}{k^{k+1}}\left(1-e^{-k v} \sum_{i=1}^{k} \frac{k^{k-i}}{(k-i) !} v^{k-i}\right)=\frac{k !}{k^{k+1}}\left(1-y^{k} \sum_{i=1}^{k} \frac{k^{k-i}}{(k-i) !} \frac{1}{v^{i}}\right) .
\end{aligned}
$$

This gives

$$
\begin{aligned}
& T(x, y)=\frac{v}{1-v} \sum_{k \geq 1}\left(\left(\frac{x}{y}\right)^{k}-x^{k} \sum_{i=1}^{k} \frac{k^{k-i}}{(k-i) !} \frac{1}{v^{i}}\right) \\
& \quad=\frac{v}{1-v}\left(\frac{x}{y-x}-\sum_{k \geq 1} x^{k}\left[\lambda^{k}\right]\left(e^{k \lambda} \frac{\lambda}{v-\lambda}\right)\right)=\frac{v}{1-v}\left(\frac{x}{y-x}-\frac{u}{v-u} \frac{1}{1-u}\right),
\end{aligned}
$$

by Lagrange's Theorem. Then

$$
T(x, y)+T(y, x)=\frac{1}{y-x}\left(\frac{x v}{1-v}-\frac{y u}{1-u}\right)=\sum_{l \geq 1} \frac{l^{l}}{l !} \frac{x y^{l}-y x^{l}}{y-x},
$$

from (9). But

$$
T_{k, m}+T_{m, k}=\left[x^{k} y^{m}\right](T(x, y)+T(y, x)),
$$

and the result follows.

We can now proceed with our verification that establishes the main result.

Proof of Theorem 1.1. Applying $z \frac{\partial}{\partial z}$ to $L(G)$, substituting for the terms that arise using Proposition 3.1, and collecting terms, we obtain

$$
\begin{aligned}
z \frac{\partial}{\partial z} L(G) & =\sum_{m \geq 1}\left(S_{m}-\frac{m^{m}}{m !}+\frac{m^{m-1}}{m !}\right) p_{m} s^{m}+\left(\frac{1}{2} \frac{(2 m)^{2 m}}{(2 m) !}-T_{m, m}\right) p_{m}^{2} s^{2 m} \\
& +\sum_{\substack{k, m \geq 1 \\
k \neq m}}\left(\frac{(k+m)^{k+m}}{(k+m) !}-T_{k, m}-T_{m, k}\right) p_{k} p_{m} s^{k+m}=0
\end{aligned}
$$

from Proposition 3.2. But, for all monomials in $F$, the exponent of $z$ equals the total degree in $\mathbf{p}$, and $F$ is the unique series in $z$ and $\mathbf{p}$ of this type whose constant term is 0 , and whose term of degree 1 in $z$ is $z p_{1}$. But $G$ is of this type, with these initial conditions, so $G$ must be the unique solution to $z \frac{\partial}{\partial z} L(G)=0$ and hence $L(G)=0$, and the result follows.

\section{Concluding Remarks}

We conclude with some observations of a more general nature, and points that deserve further consideration.

The product over parts of the partition given in the main result is reminiscent of an expression in [3] (Theorem 2.3, p. 412), as has been pointed out to us by Richard Stanley, but it is unclear whether this is a coincidence, or whether it has greater significance.

Differential equations similar to (4) can be derived for the analogous problem in which the factors are from any fixed conjugacy class, although we have not been 
able to obtain explicit solutions in any case except the class of transpositions. Such equations are special cases of the general family

$$
\frac{\partial S}{\partial t}=H\left(\left(\frac{\partial S}{\partial p_{1}}, \frac{\partial S}{\partial p_{2}}, \ldots\right), \mathbf{p}, t\right)
$$

of first order partial differential equations, where $H$ is an arbitrary function. This is called a Hamilton-Jacobi equation, and is developed by a variational argument. Thus the minimal transitive ordered factorisations of permutations into transpositions provides a combinatorial model for one equation in this family.

We can also give a character expression for $c_{\alpha}$, using a series with an extra variable $u$, whose exponent marks the number of factors in a transitive, ordered factorisation that is not necessarily minimal. Let $\tilde{\theta}=\left(\tilde{\theta}_{1}, \tilde{\theta}_{2}, \ldots\right)$ denote the conjugate of the partition $\theta=\left(\theta_{1}, \theta_{2}, \ldots\right)$. Let $\chi^{\theta}$ be the character of the ordinary irreducible representation of $\mathfrak{S}_{n}$ indexed by $\theta \vdash n$, and let $\chi_{\alpha}^{\theta}$ be the value of this character at any element of the conjugacy class $\mathcal{C}_{\alpha}$. The degree of the irreducible representation indexed by $\theta$ is $f^{\theta}$. Then, using an exponential generating function argument for connected graphs, in the context of the proof of Proposition 2.1, it is readily shown that

$$
c_{\alpha}=\left[p_{\alpha} \frac{u^{\mu(\alpha)}}{\mu(\alpha) !} \frac{z^{n}}{n !}\right] \log \left(\sum_{m \geq 0} \frac{z^{m}}{m !} \sum_{\theta \vdash m} f^{\theta} s_{\theta} e^{u \eta(\theta)}\right),
$$

where $s_{\theta}$ is the Schur symmetric function indexed by $\theta$, and (see [10] and [11], p. 64)

$$
\eta(\theta)=\left(\begin{array}{c}
n \\
2
\end{array}\right) \frac{1}{f^{\theta}} \chi_{\left[21^{n-2}\right]}^{\theta}=\sum_{i \geq 1}\left(\begin{array}{c}
\theta_{i} \\
2
\end{array}\right)-\sum_{i \geq 1}\left(\begin{array}{c}
\tilde{\theta}_{i} \\
2
\end{array}\right) .
$$

Numerical computation with this expression led us to conjecture the main result in the first place, though we have been unable to use it as the basis of a proof. Finally, we note in passing that $\eta(\theta)$ arises in the approach of Crescimanno and Taylor [1] in connection with a Casimir operator arising from a sum over representations.

\section{ACKNOWLEDGEMENTS}

This work was supported by grants from the Natural Sciences and Engineering Research Council of Canada.

\section{REFERENCES}

[1] M.Crescimanno and W.TaYlor, Large $N$ phases of chiral $Q C D_{2}$, Nuclear Phys. B 437 (1995), 3-24. MR 96g:81223

[2] J.DÉNES, The representation of a permutation as the product of a minimal number of transpositions and its connection with the theory of graphs, Publ. Math. Inst. Hungar. Acad. Sci. 4 (1959), 63-70. MR 22:6733

[3] I.M.Gel'Fand, M.M.Kapranov and A.V.Zelevinsky, "Discriminants, Resultants, and Multidimensional Determinants," Contemporary Math., Birkhäuser, Boston, 1994. MR 95e: 14045

[4] I.P.Goulden, A differential operator for symmetric functions and the combinatorics of multiplying transpositions, Trans. Amer. Math. Soc. 344 (1994), 421-440. MR 95c:20019

[5] I.P.Goulden, J. L. HARER AND D.M.JACKSON, The virtual Euler characteristic of the moduli spaces of real and complex algebraic curves (preprint).

[6] I.P.Goulden AND D.M.JACKSON, Symmetric functions and Macdonald's result for top connexion coefficients in the symmetric group, J. Algebra 166 (1994), 364-378. MR 95j:05170 
[7] I.P.Goulden AND D.M.JACKSON, "Combinatorial Enumeration," Wiley Interscience, New York, 1983. MR 84m:05002

[8] J. HARER AND D. ZAGIER, The Euler characteristic of the moduli space of curves, Invent. Math. 85 (1986), 457-485. MR 87i:32031

[9] D.M.JACKSOn, Counting cycles in permutations by group characters, with an application to a topological problem, Trans. Amer. Math. Soc. 299 (1987), 785-801. MR 88c:05011

[10] D.M.JACKSON, Some combinatorial problems associated with products of conjugacy clases of the symmetric group, J. Combin. Theory Ser. A 49 (1988), 363-369. MR 89k:05009

[11] I.G.Macdonald, "Symmetric functions and Hall polynomials," Clarendon Press, Oxford, 1981. MR 84g:05003

Department of Combinatorics and Optimization, University of Waterloo, Waterloo, Ontario, Canada N2L 3G1

E-mail address: ipgoulden@math.uwaterloo.ca

E-mail address: dmjackson@dragon.uwaterloo.ca 